\title{
Inhibition of periderm removal in all-trans retinoic acid-induced cleft palate in mice
}

\author{
YA-DONG ZHANG ${ }^{1,2}$, SHI-YI DONG ${ }^{1,2}$ and HONG-ZHANG HUANG ${ }^{1,2}$ \\ ${ }^{1}$ Guangdong Provincial Key Laboratory of Stomatology; ${ }^{2}$ Department of Oral and Maxillofacial Surgery, Guanghua \\ School of Stomatology, Hospital of Stomatology, Sun Yat-sen University, Guangzhou, Guangdong 510055, P.R. China
}

Received August 21, 2015; Accepted February 1, 2017

DOI: $10.3892 /$ etm.2017.4938

\begin{abstract}
Cleft palate is a common craniofacial birth defect. The aim of the present study was to investigate the effect of excess all-trans retinoic acid (atRA) on periderm removal and the disappearance of basal medial edge epithelial (MEE) cells during palatogenesis, particularly during the stage prior to contact. atRA $(200 \mathrm{mg} / \mathrm{kg})$ was administered to C57BL $/ 6 \mathrm{~N}$ mice at embryonic day (E) 12.0 by gavage. Fetal palates were processed and analyzed by histology and electron microscopy. Single palate shelf peridermal cells were removed and cultured in the presence of atRA $(3 \mu \mathrm{M})$ only or in the presence of or the caspase inhibitor, Z-VAD $(100 \mu \mathrm{M})$ only, for $48 \mathrm{~h}$. Once cultured, morphological changes were analyzed by histological staining and electron microscopy. A TUNEL assay was used to detect apoptotic neurons. Paired palatal shelves with periderm removal were cultured in the presence of atRA $(3 \mu \mathrm{M})$ only or in the presence of Z-VAD $(100 \mu \mathrm{M})$ only for $48 \mathrm{~h}$ and analyzed by hematoxylin and eosin staining. At E14.5, medial edge epithelium periderm was retained in the atRA-treated palates but had been shed prior to contact in the control groups. In addition, atRA was revealed to disrupt the cell cycle in the periderm by downregulating $\mathrm{p} 21$. Furthermore, atRA inhibited apoptosis in the periderm and basal MEE cells; however, atRA exhibited no effect on basement membrane degradation in single palatal organ culture. Additionally, once paired palates were cultured for $48 \mathrm{~h}$, all of the groups in which the periderm had been removed exhibited confluence of the embryonic palatal mesenchyme. The present results suggest that periderm removal is inhibited in atRA-induced cleft palate in mice and that removal of the periderm contributes to EPM confluence in vitro.
\end{abstract}

Correspondence to: Dr Hong-Zhang Huang, Department of Oral and Maxillofacial Surgery, Guanghua School of Stomatology, Hospital of Stomatology, Sun Yat-sen University, 74 Zhongshan Second Road, Guangzhou, Guangdong 510055, P.R. China E-mail: hhzhuanghongzhang@163.com

Key words: periderm, all-trans retinoic acid, p21, cleft palate

\section{Introduction}

Cleft palate is one of the most common craniofacial birth defects (1). The development of the second palate requires several steps, including the growth, reorientation and fusion of the palate shelves (2). The medial edge epithelium is composed of two layers of cell: Basal cells and peridermal cells. The paired shelves meet at the middle edge to form the midline epithelial seam (MES). Simultaneously, the MES rapidly disappears and a continuous plate forms that separates the nasal and oral cavities. Interruption of any of the processes during palatogenesis can result in cleft palate (3).

During palatogenesis, the periderm covers the MEE cells that are sloughed off prior to palate contact (3). Subsequently, firm contact is established with the basal MEE cells and the MES is formed. A recent study has proposed that programmed cell death is the major fate of peridermal cells (4). However, the predominant cause of periderm removal in the region of the MEE cells remains unclear.

On a mechanistic level, transforming growth factor beta-3 (TGF- $\beta_{3}$ ) is considered to be one of the essential factors facilitating periderm removal based on its expression $(5,6)$. It has been recently reported that $T G F-\beta 3$ regulates removal of the periderm through $\Delta \mathrm{Np} 63$ during palatogenesis (7). $T G F-\beta 3$, which has an essential role in palatogenesis, has been studied intensively (8). It has been proposed that the MES independently yet sequentially undergoes cell cycle arrest, cell migration and apoptosis, following the treatment of isolated MES cells with TGF- $\beta 3$ (9). Recent studies have demonstrated that TGF- $\beta$ mediated by IRF6 is necessary for palatal fusion in mice and that TGF- $\beta$ signaling is mediated by Tak1, Smad 4 and Trim33 during development of the palate $(10,11)$.

Retinoic acid (RA) is a vitamin A-derived morphogen that is important for organ development in vertebrates (12). $\mathrm{RA}$ is a strong teratogen that can induce various types of cleft palate based on the intragastric dose and stage at which it is administered in pregnant mice $(13,14)$. For example, atRA exposure on gestation day (GD) 10 induces cleft palate with small palatal shelves that fail to elevate and MEE cells that differentiate into squamous epithelium, whereas atRA exposure on GD12 results in normal-sized palatal shelves that are able to contact one another; however they never fuse and MEE cells differentiate into ciliated epithelium $(13,14)$. It has been previously reported that atRA inhibits embryonic palatal 
mesenchymal (EPM) proliferation by upregulating p21, which prevents G1/S progression (15).

The purpose of the present study was to analyze the effects of excess atRA on periderm removal and basal MEE cell disappearance during palatogenesis using palate organ culture, scanning electron microscopy (SEM), transmission electron microscopy (TEM), histological analysis and TUNEL assay.

\section{Materials and methods}

Mice. A total of $66 \mathrm{C} 57 \mathrm{BL} / 6 \mathrm{~N}$ mice were used in the present study. All animals used were purchased from Sun Yat-sen University (Guangzhou, China). Female C57BL/6 mice (age, 10-12 weeks; weight, 25-30 g) were mated with mature males (age, 7-8 weeks; weight, 18-25 g) between 8 and 10 pm. All animals were housed at a temperature of $28^{\circ} \mathrm{C}$ and a humidity of $50 \%$ with a $12 \mathrm{~h}$ light/dark cycle and were provided with food pellets and tap water ad libitum. The gender ratio of total mice was 1:5 (female to male). The detection of a vaginal plug was denoted as gestation day 0 (GD 0). Pregnant females were administered a single dose of all-trans RA $(200 \mathrm{mg} / \mathrm{kg})$, which had been previously dissolved in corn oil $(20 \mathrm{mg} / \mathrm{ml})$, by gavage (Sigma-Aldrich; Merck Millipore, Darmstadt, Germany). Control animals were given the equivalent volume of corn oil alone. Pregnant females were sacrificed by cervical dislocation on embryonic day (E) 13.5, E14.5 and E15.5. Once soaked in $90 \%$ alcohol for $1 \mathrm{~min}$, the fetuses were exteriorized from the uterus under aseptic conditions. All animal experiments conducted in the present study have been reviewed and approved by the Institutional Animal Care and Use Committee, Sun Yat-Sen University (DB-15-0302).

Periderm cell removal. The periderm cell removal method was developed based on the report by Cuervo and Covarrubias (16). Palates were dissected and incubated in $0.25 \%$ trypsin with ethylenediaminetetraacetic acid (Invitrogen; Thermo Fisher Scientific Inc., Waltham, MA, USA) at $4^{\circ} \mathrm{C}$ for $5 \mathrm{~min}$. The palate medial edges were washed until the periderm cell layer sloughed off. Subsequently, the palates were washed and incubated in Dulbecco's modified Eagle medium supplemented with $10 \%$ fetal bovine serum (Gibco; Thermo Fisher Scientific, Inc.) for $10 \mathrm{~min}$ to terminate the digestion of trypsin. The same procedure was performed on control palates that did not contain trypsin.

Palate organ culture. E13.5 fetuses were used for the palate organ culture. One side of the palatal shelf was gently removed from the maxilla in cold Hanks buffer (Invitrogen; Thermo Fisher Scientific Inc.). The dissected palatal shelf was subsequently placed on MF-Millipore membranes (Merck Millipore) that had been pre-treated with boiling water and washed with $75 \%$ ethanol. A single palatal shelf was placed on an autoclaved grille with the oral side facing upward in an organ culture dish. BGJb medium (Invitrogen; Thermo Fisher Scientific, Inc.) supplemented with ascorbic acid $(0.1 \mu \mathrm{g} / \mathrm{ml}$; Sigma-Aldrich; Merck Millipore) was used in the culture system. Retinoic acid ( $3 \mu \mathrm{M}$; Sigma-Aldrich; Merck Millipore) or Z-VAD (100 $\mu \mathrm{M}$; Beyotime Institute of Biotechnology, Jiangsu, China) were added directly to the medium. Control palates were collected from E13.5 fetuses and cultured in BGJb medium with ascorbic acid only. All palatal shelves were cultured at $37^{\circ} \mathrm{C}$ in an atmosphere containing $5 \% \mathrm{CO}_{2}$ and $95 \%$ air for $48 \mathrm{~h}$. The medium was changed every $24 \mathrm{~h}$. Following culturing, the harvested palatal shelves were fixed in $4 \%$ paraformaldehyde in $0.1 \mathrm{M}$ phosphate buffer ( $\mathrm{pH} \mathrm{7.4)}$ at room temperature for $24 \mathrm{~h}$, embedded in paraffin and sliced to generate $3-\mu$ m-thick serial sections. For the culture of paired palatal shelves, two palatal shelves were forced to contact one another at the medial edges and processed as described for the single palatal shelf culture.

SEM. Harvested palatal shelves were fixed in $2.5 \%$ glutaraldehyde in $0.1 \mathrm{M}$ sodium cacodylate ( $\mathrm{pH} 7.4$ ) for $12 \mathrm{~h}$ and post-fixed in $1 \%$ osmium tetroxide for $2 \mathrm{~h}$. Subsequently, palatal shelves were dehydrated in an ethanol series, dried with liquid $\mathrm{CO}_{2}$ and coated with a thin layer of gold. Samples were observed using a Philips Quanta 400 (Philips Medical Systems B.V., Eindhoven, Netherlands) scanning electron microscope (magnification, x200 and x1,500).

TEM. Palatal shelves were harvested in vivo following culturing for $48 \mathrm{~h}$. Samples were fixed in $2.5 \%$ glutaraldehyde and $2 \%$ paraformaldehyde in $0.1 \mathrm{M}$ cacodylate buffer $(\mathrm{pH} 7.4)$ for $2 \mathrm{~h}$ at room temperature. Subsequently, the samples were rinsed several times in cacodylate buffer and post-fixed in $1 \%$ osmium tetroxide for $1 \mathrm{~h}$. Once the shelves were embedded in resin, semi-thin sections $(0.5 \mu \mathrm{m})$ were cut to locate the MEE area and then trimmed to generate ultrathin sections $(100 \mathrm{~nm})$. Ultrathin sections were stained with lead citrate and uranyl acetate prior to observation, using a Tecnai G2 Spirit Twin (FEI, Hillsboro, OR, USA) transmission electron microscope. (magnification, x3,900).

Histological analysis and TUNEL assay. Immunohistochemistry (IHC) was performed according to standard protocols. Cultured palates were fixed in $4 \%$ paraformaldehyde for $24 \mathrm{~h}$ at room temperature, embedded in paraffin and sliced to generate $3-\mu$ m-thick serial sections. The sections were deparaffinized in xylene and rehydrated using a gradient of alcohol. Rabbit polyclonal antibodies were used as primary antibodies for p21 (ab109199; 1:250; Abcam, Cambridge, UK) staining. A two-step IHC detection reagent kit (SPN9001; ZSGB-BIO, Beijing, China) was used to localize the primary antibody. Subsequently, all the sections were treated with 3,3'-diaminobenzidine (ZSGB-BIO) under the microscope and stained with hematoxylin (ZSGB-BIO) for $30 \mathrm{sec}$. TUNEL assay was performed using a commercial kit (TUN11684817; Roche Diagnostics GmbH, Mannheim, Germany) according to standard procedure and the nuclei were stained with 3,3'-diaminobenzidine (ZSGB-BIO). All of the sections were viewed directly using an Axioskop 40 (Carl Zeiss AG, Oberkochen, Germany) microscope (magnification, $\mathrm{x} 10$ and $\mathrm{x} 20$ ).

Statistical analysis. All experiments were performed at least three times. Data was analyzed using SPSS software (13.0; SPSS Inc., Chicago, IL, USA) with Student's t-test. Data was expressed as mean \pm standard deviation. $\mathrm{P}<0.05$ was considered to indicate a statistically significant difference. 


\section{Results}

Hematoxylin and eosin $(H \& E)$ observations. atRA exposure on GD12.0 induced cleft palate in $75 \%$ of the fetuses $(n=100)$, whereas no abnormal fetuses were observed in the control group $(n=80)$. H\&E staining revealed a firm palate contact was established at the medial edge and a bilayer was formed at the MES in the control palate at E14.5 (Fig. 1A). Conversely, peridermal cells were exhibited in the medial edge epithelium in atRA-treated palates during the approximate time of contact between opposing palates at E14.5 (Fig. 1B). At E15.5, the MES bilayer had disappeared and confluence of the EPM was exhibited in the control palate (Fig. 1C). However, in atRA-exposed fetuses, gaps remained between the palatal shelves and a cleft palate developed (Fig. 1D).

SEM and TEM observations. To observe the morphological changes in the MEE periderm in atRA-treated palates, the palate shelves were observed by SEM and TEM. The sloughing peridermal cells were observed on the surface of the medial edge epithelium in the control groups (Fig. 2A). Conversely, almost no sloughing cells were observed on the surface of the medial edge epithelium in the atRA-treated palates (Fig. 2B). Furthermore, lateral cell-to-cell contacts were lost between the detected peridermal and basal cells (Fig. 2C) and tight junctions were apparent between the peridermal and basal cells (Fig. 2D).

Protein expression levels of 21 in the periderm of the medial edge epithelium. To examine whether the G1/S checkpoint was disrupted by atRA treatment, IHC was performed (Fig. 3A). As one of the regulators of G1 to $S$ progression, p21 is an important cyclin-dependent kinase inhibitor (15). At E13.5, the levels of p21 protein expression in the peridermal cells did not significantly differ between atRA-treated palates and control palatal shelves $(\mathrm{P}=0.64$; Fig. $3 \mathrm{~B})$. Prior to contact of the palatal shelves at E14.5, p21 protein was expressed at a significantly decreased level in the atRA-treated palatal periderm when compared with the control palatal periderm $(\mathrm{P}<0.05$; Fig. $3 \mathrm{~B})$. However, at E15.5, the level of p21 protein expression was significantly increased in the atRA-treated palatal periderm when compared with the control group $(\mathrm{P}<0.05$; Fig. 3B).

atRA inhibits periderm removal in vitro by inhibiting apoptotic cell death in peridermal cells. To investigate whether peridermal cells were affected by atRA in vitro and whether basal cells were affected by atRA when the periderm was artificially removed (Fig. 4), a single palatal shelf with the periderm removed was cultured in the presence of atRA or Z-VAD. Following culturing for $48 \mathrm{~h}$, the medial edge epithelium in control palates was not present, despite periderm removal (Fig. 5). In the presence of atRA or Z-VAD, MEE cells were retained in the untreated periderm groups, whereas the majority of the basal MEE cells remained on the palate surface and exhibited basement membrane degradation in groups where the periderm was removed. TUNEL assay was utilized to assess whether apoptosis was affected by atRA (Fig. 6A). In the untreated periderm groups, the proportion of apoptotic cells significantly decreased in the atRA and the Z-VAD-treated groups when compared with the control

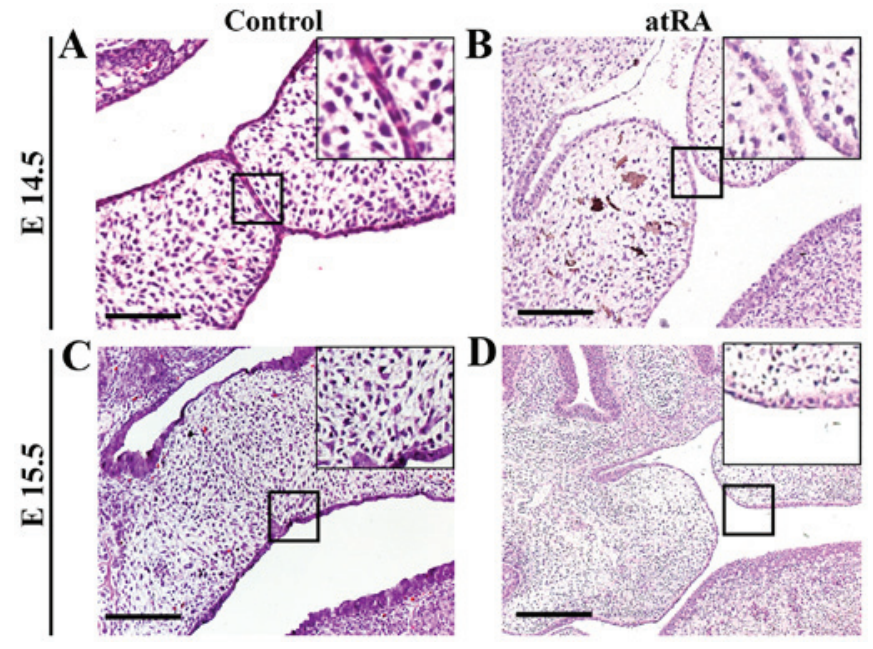

Figure 1. Hematoxylin and eosin staining indicated the development of the palate shelf at E14.5 and E15.5 in the atRA-treated and control groups. (A) Palatal shelf contact was exhibited at E14.5 in the control group. (B) Palates were observed to be sliding off one another at E14.5 in the atRA-treated group. (C) Palate fusion at E15.5 was exhibited in the control group. (D) Development of cleft palate in the atRA-treated groups was observed at E15.5. Scale bar, $20 \mu \mathrm{m}$. Magnification, x20. atRA, all-trans retinoic acid; E, embryonic day.
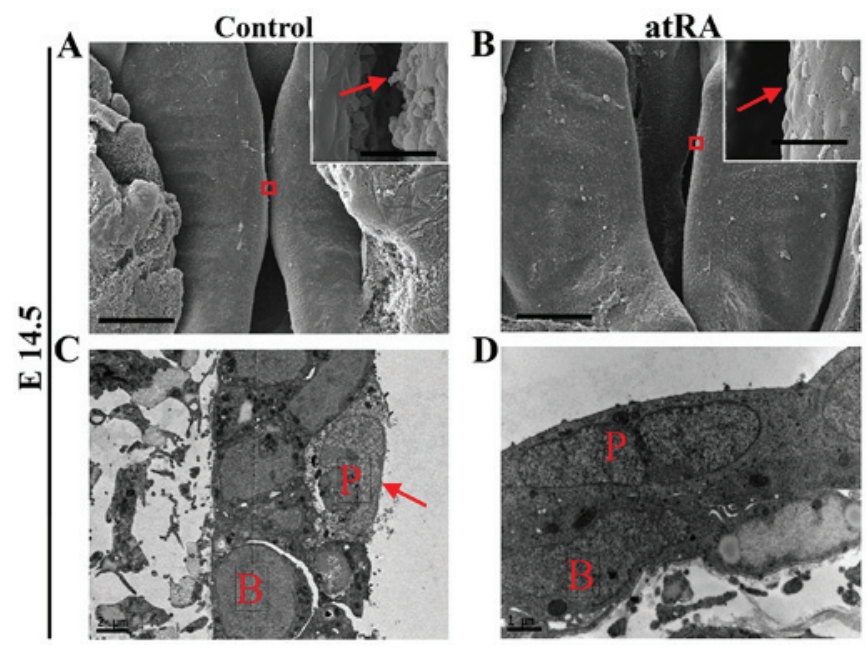

Figure 2. Scanning electron microscopy and transmission electron microscopy of morphological changes in the MEE periderm in atRA-treated and control palates at E14.5. (A) Sloughing off of the periderm on the surface of the medial edge epithelium in the control groups (arrow). Scale bar, $1 \mathrm{~mm}$. (B) The periderm on the surface of the medial edge epithelium was intact in atRA-treated palatal shelves (arrow). Scale bar, $1 \mathrm{~mm}$. (C) Apoptotic peridermal cell (arrow) and loss of the junction between the peridermal and basal cells. Scale bar, $2 \mu \mathrm{m}$. (D) Tight junctions between peridermal cells and basal cells. Scale bar, $1 \mu \mathrm{m}$ (A, B). atRA, all-trans retinoic acid; E, embryonic day; MEE, medial edge epithelial; P, peridermal cell; B, basal cell.

groups $(\mathrm{P}<0.05$; Fig. 6B). Similarly, the groups that underwent periderm removal exhibited significantly decreased numbers of TUNEL-positive cells in the atRA or Z-VAD-treated groups when compared with the control groups $(\mathrm{P}<0.05$; Fig. 6B).

Periderm removal contributes to EPM confluence in vitro. As the removal of the periderm in single palate cultures resulted in basement membrane degradation in the presence of atRA or Z-VAD, a paired palate culture was constructed 

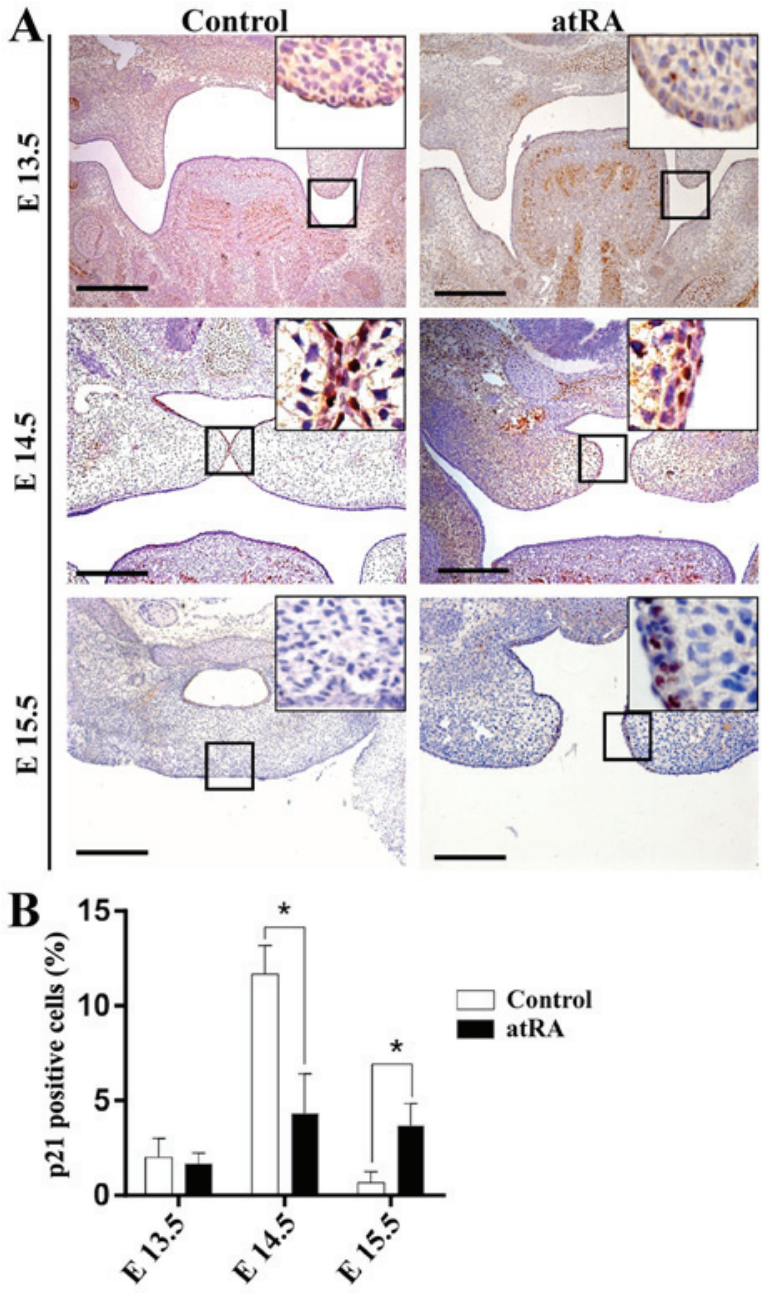

Figure 3. Immunohistochemical staining for P21. (A) Protein expression levels of P21 in the peridermal cells of MEE in control palatal shelves and atRA-treated palates at E13.5, E14.5 and E15.5. Scale bar, 50 $\mu \mathrm{m}$. (B) At E13.5, the protein expression levels of $\mathrm{P} 21$ in peridermal cells did not significantly differ between atRA-treated palates and control palatal shelves $(\mathrm{P}=0.64)$. At E14.5, P21 protein was expressed at a significantly decreased level in the atRA-treated palatal periderm in comparison to the control palatal periderm. At E15.5, the protein expression level of p21 was significantly increased in the atRA-treated palatal periderm compared with the control groups. Error bars represent the mean \pm standard deviation. ${ }^{*} \mathrm{P}<0.05$. Scale bar, $50 \mu \mathrm{m}$. Magnification, x10. atRA, all-trans retinoic acid; MEE, medial edge epithelial; E, embryonic day.

to determine whether periderm removal contributed to EPM confluence in vitro. The success rate is presented in Table I. In the control group, no MES were observed and the palates fused completely (Fig. 7A). Conversely, EPM was separated by the thick MES barrier in the untreated periderm group in the presence of atRA (Fig. 7B). Following $48 \mathrm{~h}$ in culture, no MES fracture was observed in the untreated periderm group in the presence of Z-VAD (Fig. 7C). Following periderm removal, no MES was observed after culture for $48 \mathrm{~h}$ (Fig. 7D). Following culturing of the paired palates for $48 \mathrm{~h}$ in the presence of $3 \mu \mathrm{M}$ atRA, the EPM broke through the MES barrier in the groups that underwent periderm removal (Fig. 7E). Moreover, paired palates were processed to remove the periderm and cultured in the presence of Z-VAD, the point of fracture in the MES appeared in the groups with periderm removal (Fig. 7F).
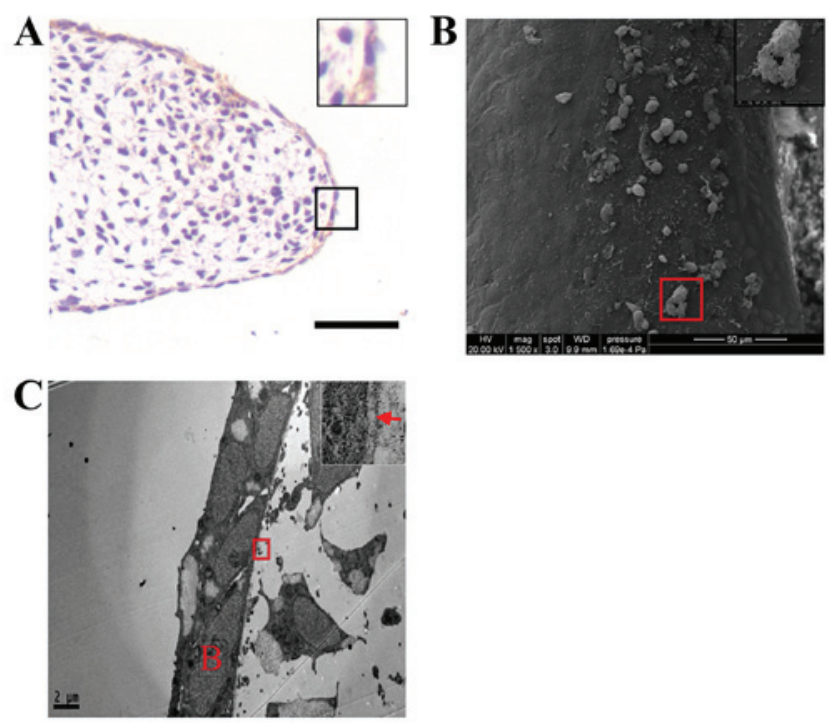

Figure 4. Hematoxylin and eosin staining, transmission electron microscopy and scanning electron microscopy observations of periderm removal in the single palatal shelf. (A) Once the periderm was removed, the medial edge epithelium exhibited a single layer of basal cells. Scale bar, $20 \mu \mathrm{m}$. (B) Scanning electron microscopy revealed sloughing of the peridermal cells. (C) Transmission electron microscopy revealed a single layer of basal cells with an intact basement membrane (arrow). B, basal cell.
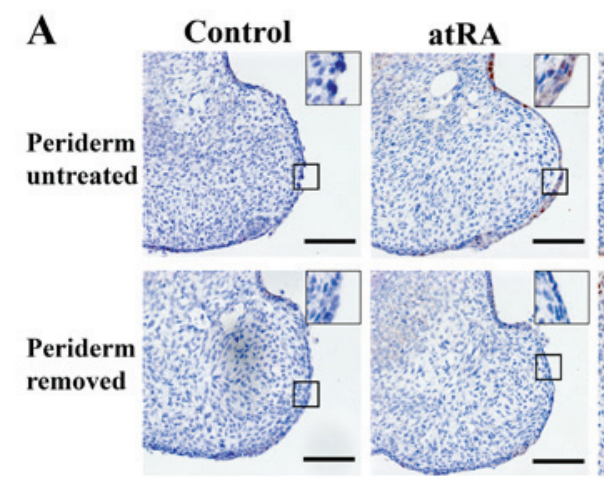

Z-VAD
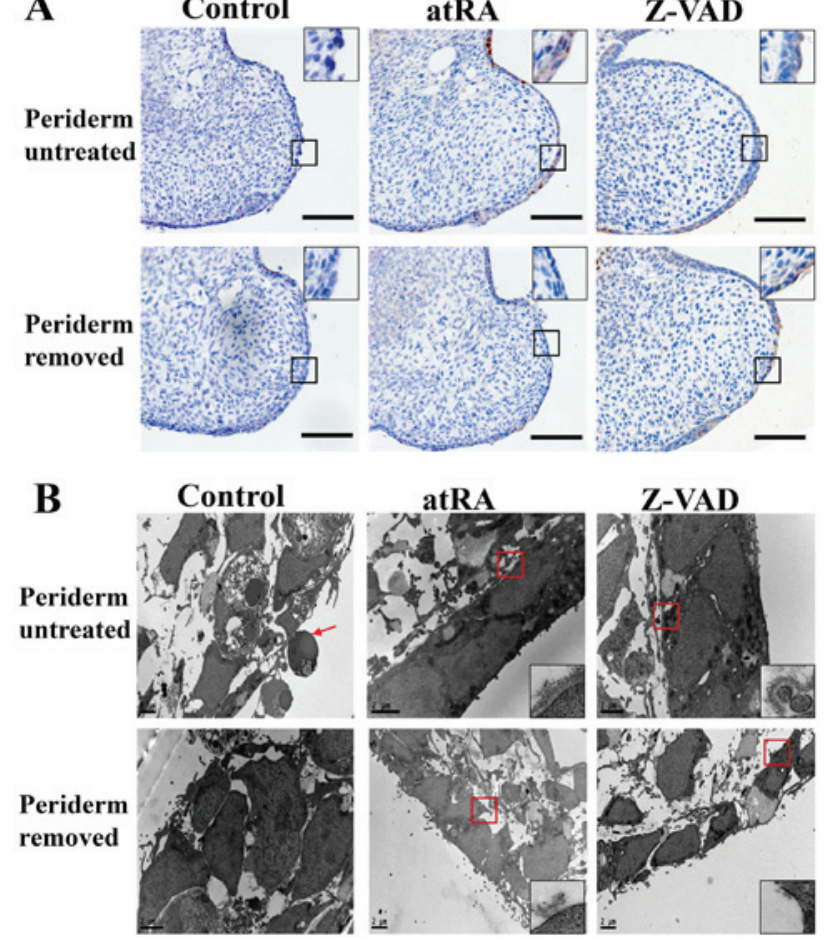

Figure 5. Immunohistochemistry staining of P21 and transmission electron microscopy observations of differences in the medial edge epithelium in a single palate culture for $48 \mathrm{~h}$ with or without periderm removal. (A) The medial edge epithelium disappeared in the control palates and the MEE cells were retarded despite periderm removal when they were cultured with atRA or Z-VAD. Scale bar, $20 \mu \mathrm{m}$. (B) The medial edge epithelium and basement disappeared in control palates despite periderm removal. In untreated periderm groups, basal and peridermal cells were intact in the presence of atRA or Z-VAD; however, in the groups with periderm removal, most of the basal cells remained on the surface of the palate and exhibited basement membrane degradation. Arrow in B indicates apoptotic cell. All of the experiments were repeated at least three times. Scale bar, $2 \mu \mathrm{m}$. Magnification, x20. MEE, medial edge epithelial; atRA, all-trans retinoic acid. 
Table I. Success rate of paired palatal shelves organ culture.

\begin{tabular}{lcr} 
Periderm treatment and culture method & Successful (EPM confluence) & Failed (EPM sep \\
\hline Periderm untreated & $21 / 25(84)$ & $4 / 25(16)$ \\
Periderm untreated + atRA & $6 / 25(24$ & $19 / 25(76)$ \\
Periderm untreated + Z-VAD & $2 / 20(10)$ & $18 / 20(90)$ \\
Periderm removed & $18 / 20(90)$ & $2 / 20(10)$ \\
Periderm removed + atRA & $9 / 10(90)$ & $1 / 10(10)$ \\
Periderm removed + Z-VAD & $8 / 10(80)$ & $2 / 10(20)$
\end{tabular}

The criterion that determined the success rate of different culture methods was based on histology staining. Data are presented as $\mathrm{n} / \mathrm{N}(\%)$. atRA, all-trans retinoic acid; EPM, embryonic palatal mesenchyme.

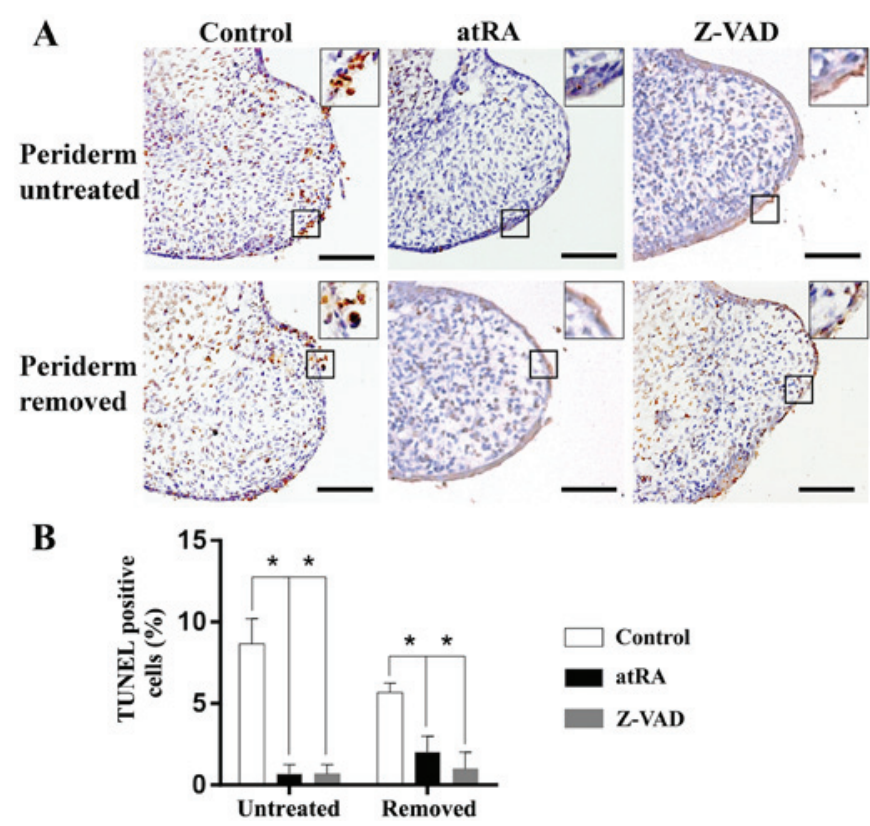

Figure 6. TUNEL assay in single palate cultures for $48 \mathrm{~h}$ with or without periderm removal. (A) TUNEL-positive cells were detected in the control groups with or without periderm removal. Following treatment with atRA or $\mathrm{Z}$-VAD, the number of apoptotic cells decreased in the untreated periderm groups and groups with periderm removal. Scale bar, $20 \mu \mathrm{m}$. (B) The ratio of apoptotic cells was significantly higher in the control groups when compared with the atRA or Z-VAD-treated groups $(\mathrm{P}<0.05)$ irrespective of whether the periderm was removed. Error bars represent the mean \pm standard deviation. ${ }^{*} \mathrm{P}<0.05$. atRA, all-trans retinoic acid.

\section{Discussion}

During fusion of the palate, persistence of the periderm resulted in poor adhesion between the opposing MEE cells, resulting in cleft palate formation with continuous maxillary growth (7). In the present study, murine models of atRA-exposed embryos resulted in cleft palate. Notably, large and small gaps were consistently observed between the opposing palatal shelves, indicating that the proper connection and fusion of the palate contributed to the expansion of the palate volume.

Sloughing peridermal cells have been observed prior to palatal adhesion of the opposing MEE cells during normal palatogenesis (17). Furthermore, it has been reported that atRA induces apoptosis in MEE cells following RA
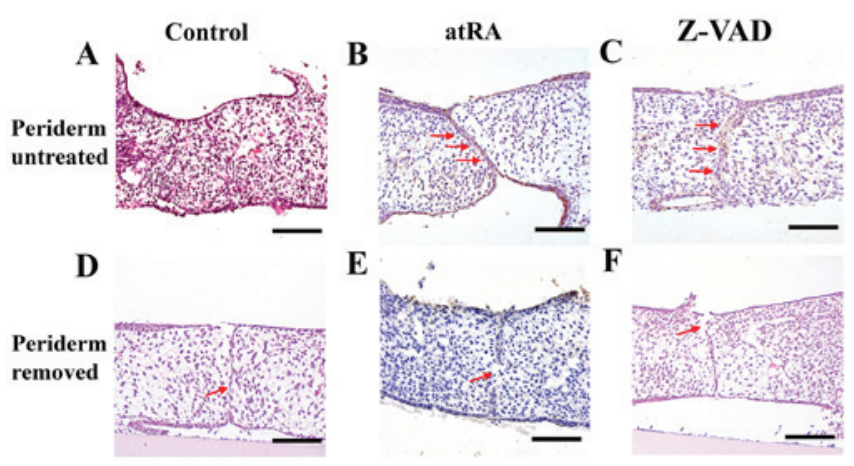

Figure 7. Culture of paired palatal shelves for $48 \mathrm{~h}$. Complete fusion of the palates occurred in the (A-C) untreated periderm groups and (D-F) in the groups with periderm removal. (A) No MES were observed and the palate fused completely in the control group with untreated periderm. (B) EPM was separated by the thick MES barrier in the untreated periderm group in the presence of atRA $(3 \mu \mathrm{M})$. (C) In the presence of Z-VAD $(100 \mu \mathrm{M})$, EPM was separated by the multilayer of the MES barrier in the untreated periderm groups. (D) The medial edge epithelium broke apart into epithelial islands in the groups with periderm removal. (E) After culturing the paired palates in the presence of atRA $(3 \mu \mathrm{M})$, two cell layers of MES were observed in the untreated periderm groups. (F) The MES became disrupted (arrow) in the groups with periderm removal that were treated with Z-VAD $(100 \mu \mathrm{M})$. Furthermore, the MES (arrow) was disrupted in all groups that were subjected to periderm removal (D, E and F). Scale bar, $20 \mu \mathrm{m}$. Magnification, x20. atRA, all-trans retinoic acid; EPM, embryonic palatal mesenchyme; MES, midline epithelial seam.

treatment in pregnant mice at E11.5, with small palatal shelves that failed to elevate (18). In the present study, almost no sloughing cells were observed on the surface of the medial edge epithelium at E14.5 in pregnant mice that were treated with atRA at E12.0.

RA is known to prevent cell cycle progression from G1 to $\mathrm{S}$ in EPM cells (19). As one of the regulators of the progression from G1 to $\mathrm{S}, \mathrm{p} 21$ is an important cyclin-dependent kinase inhibitor (15). In a previous study, the upregulation of p21 was detected in EPM palates following treatment with atRA in pregnant mice at E10.0; however, no apparent change was observed in EPM palates in pregnant mice treated at E12.0 (15). In the present study, the level of p21 protein expression was downregulated in the atRA-treated palatal periderm when compared with the control palatal periderm at E14.5. However, following culture for $48 \mathrm{~h}$, almost no p21 positive cells were detected in MEE cells. The possible reasons for 
this are the environmental differences between in vivo and in vitro models. High concentrations of RA are able to induce apoptosis and therefore this approach has been widely used to induce apoptosis in MEE cells in vitro (20). Conversely, low concentrations of RA, such as $0.1 \mu \mathrm{M}$ of $\mathrm{RA}$, are able to promote epithelial cell proliferation (21). In the present study, a final atRA concentration of $3 \mu \mathrm{M}$ was used in the culture medium (data not shown).

The ability of periderm removal to accelerate basal cell disappearance has not been frequently reported during palatogenesis. As a barrier between adjacent organs, a deficiency in the periderm may result in premature fusion between the palate and tongue or oral mucosa, resulting in cleft palate (22). During palatogenesis in Jag $2^{\Delta \mathrm{DSL} / \Delta \mathrm{DSL}}$ mutants, fusion of the bilateral palate with the tongue was exhibited and the majority of the newly formed palate-tongue seam disappeared (23). In the present study, the periderm was removed artificially. Following culturing of the single palatal shelf with exposed basal cells in the presence of atRA or Z-VAD for $48 \mathrm{~h}$, the majority of the basal MEE cells were retained and exhibited basement membrane degradation. Results of the TUNEL assay indicated that atRA inhibited MEE cell apoptosis and basement membrane degradation in the presence of the periderm; however, atRA exhibited no effect on the progression of basement membrane degradation. It was concluded that cell death was not the only cause of basement membrane disappearance in vitro.

The present study evaluated single palatal shelf cultures to eliminate interference from the opposing palatal shelf and to examine the morphological changes in peridermal cells and basal cells by TEM. It should be stressed that not all developmental processes in culture mimic those in vivo and the separation of tissues from their natural surroundings increases the probability of aberrant cell behavior. However, our study may provide a novel understanding of MEE cell disappearance during palatogenesis. Thus, further advanced research methods and additional research focused on mechanism should be conducted in the future.

\section{Acknowledgements}

The present study was supported by the Natural Science Foundation of China, (grant no. 81300862), the Project of Health and Family Planning Commission of Shenzhen Municipality (grant no. 201302202) and the Natural Science Foundation of Guangdong Province (grant no. S2011040004190).

\section{References}

1. Chai Y and Maxson RJ: Recent advances in craniofacial morphogenesis. Dev Dyn 235: 2353-2375, 2006.

2. Bush JO and Jiang R: Palatogenesis: Morphogenetic and molecular mechanisms of secondary palate development. Development 139: 231-243, 2012.
3. Nawshad A: Palatal seam disintegration: To die or not to die? that is no longer the question. Dev Dyn 237: 2643-2656, 2008.

4. Fitchett JE and Hay ED: Medial edge epithelium transforms to mesenchyme after embryonic palatal shelves fuse. Dev Biol 131: 455-474, 1989.

5. Gehris AL, Pisano MM, Nugent $P$ and Greene RM: Regulation of TGF beta 3 gene expression in embryonic palatal tissue. In Vitro Cell Dev Biol Anim 30A: 671-679, 1994.

6. Fitzpatrick DR, Denhez F, Kondaiah P and Akhurst RJ: Differential expression of TGF beta isoforms in murine palatogenesis. Development 109: 585-595, 1990.

7. Hu L, Liu J, Li Z, Ozturk F, Gurumurthy C, Romano RA, Sinha S and Nawshad A: TGF $\beta 3$ regulates periderm removal through $\Delta$ Np63 in the developing palate. J Cell Physiol 230: 1212-1225, 2015.

8. Kaartinen V, Voncken JW, Shuler C, Warburton D, Bu D, Heisterkamp N and Groffen J: Abnormal lung development and cleft palate in mice lacking TGF-beta 3 indicates defects of epithelial-mesenchymal interaction. Nat Genet 11: 415-421, 1995.

9. Nawshad A, LaGamba D and Hay ED: Transforming growth factor beta (TGFbeta) signalling in palatal growth, apoptosis and epithelial mesenchymal transformation (EMT). Arch Oral Biol 49: 675-689, 2004.

10. Lane J, Yumoto K, Azhar M, Ninomiya-Tsuji J, Inagaki M, Hu Y, Deng CX, Kim J, Mishina Y and Kaartinen V: Tak1, Smad4 and Trim 33 redundantly mediate TGF- $\beta 3$ signaling during palate development. Dev Biol 398: 231-241, 2015.

11. Iwata J, Suzuki A, Pelikan RC, Ho TV, Sanchez-Lara PA, Urata M, Dixon MJ and Chai Y: Smad4-Irf6 genetic interaction and TGF $\beta$-mediated IRF6 signaling cascade are crucial for palatal fusion in mice. Development 140: 1220-1230, 2013.

12. Ross SA, McCaffery PJ, Drager UC and De Luca LM: Retinoids in embryonal development. Physiol Rev 80: 1021-1054, 2000.

13. Birnbaum LS, Harris MW, Stocking LM, Clark AM and Morrissey RE: Retinoic acid and 2,3,7,8-tetrachlorodibenzo-p-dioxin selectively enhance teratogenesis in C57BL/6N mice. Toxicol Appl Pharmacol 98: 487-500, 1989.

14. Abbott BD, Harris MW and Birnbaum LS: Etiology of retinoic acid-induced cleft palate varies with the embryonic stage. Teratology 40: 533-553, 1989.

15. Wang M, Huang $\mathrm{H}$ and Chen $\mathrm{Y}: \mathrm{Smad} 2 / 3$ is involved in growth inhibition of mouse embryonic palate mesenchymal cells induced by all-trans retinoic acid. Birth Defects Res A Clin Mol Teratol 85: 780-790, 2009.

16. Cuervo R and Covarrubias L: Death is the major fate of medial edge epithelial cells and the cause of basal lamina degradation during palatogenesis. Development 131: 15-24, 2004.

17. Martínez-Alvarez C, Tudela C, Pérez-Miguelsanz J, O'Kane S, Puerta $\mathrm{J}$ and Ferguson MW: Medial edge epithelial cell fate during palatal fusion. Dev Biol 220: 343-357, 2000.

18. Okano J, Suzuki S and Shiota K: Involvement of apoptotic cell death and cell cycle perturbation in retinoic acid-induced cleft palate in mice. Toxicol Appl Pharmacol 221: 42-56, 2007.

19. Yu Z, Lin J, Xiao Y, Han J, Zhang X, Jia H, Tang Y and Li Y: Induction of cell-cycle arrest by all-trans retinoic acid in mouse embryonic palatal mesenchymal (MEPM) cells. Toxicol Sci 83: 349-354, 2005.

20. Cuervo R, Valencia C, Chandraratna RA and Covarrubias L: Programmed cell death is required for palate shelf fusion and is regulated by retinoic acid. Dev Biol 245: 145-156, 2002.

21. Bamberger C, Pollet D and Schmale H: Retinoic acid inhibits downregulation of DeltaNp63alpha expression during terminal differentiation of human primary keratinocytes. J Invest Dermatol 118: 133-138, 2002.

22. Kurosaka H, Iulianella A, Williams T and Trainor PA: Disrupting hedgehog and WNT signaling interactions promotes cleft lip pathogenesis. J Clin Invest 124: 1660-1671, 2014.

23. Casey LM, Lan Y, Cho ES, Maltby KM, Gridley T and Jiang R: Jag2-Notch1 signaling regulates oral epithelial differentiation and palate development. Dev Dyn 235: 1830-1844, 2006. 